\title{
A Novel Ear Identification System for Security Applications
}

\author{
S. Tayyaba, M. W. Ashraf, N. Afzulpurkar, A. Hussain, M. Imran, and A. Noreen
}

\begin{abstract}
In this paper, a novel ear identification system for security applications is presented. The structure of human ear is an inimitable characteristic that remains comparatively unchanged with time. The consistent and stout features of ear can be extracted from a distance. The proposed system consists of three modules that are database module, image processing module and identification module. The system takes ear images and extracts useful information by image processing module. The resultant information and templates are stored in data base. The suspect's image after processing by image processing module is sent to identification module for assessment with the record present in database using template matching. Then the identification module makes decision regarding similarity or difference between the processed images. Finally, the decision is sent to report generator component that generates the final report. The system has been tested for 24 ear images by using templates. The results show that the system is $98 \%$ accurate.
\end{abstract}

Index Terms-Database module, ear identification, image processing module, security applications, template matching.

\section{INTRODUCTION}

Ear-base identification is one of the emerging biometric techniques for security applications. This identification method is very useful in small towns and remote areas where robbery is very common and increasing day by day. In majority of robberies, the criminals wear a cap, wrap and a cloth hanging from the cap to their face thus cover up their faces. However, the ears of the criminal remain detectable. On this base, the criminals can be identified. In general, it is predicted that the ear shape of any person is unique and distinctive. Although, humans cannot discriminate other people on the basis of their ears, but an ear identification system can identify, extract and differentiate between the inimitable features of ears. Basically the human ears can only be used in automated and programmed identification with reliability. Ear identification is more consistent as compared to face recognition. The inconsistency in face recognition is due to its appearance, direction and aging effects. The location of ear on head side makes the identification process easy and simple.In 1949, Alfred Iannarelli was the first one who used ear features to recognize people. He physically calculated the distances between various parts of ear and

Manuscript received August 20, 2012; revised October 10, 2012

Shahzadi Tayyaba, Nitin Afzulpurkar, and Akhtar Husain are with the School of Engineering and Technology, Asian Institute of Technology (AIT), Bangkok, Thailand (e-mail: Shahzadi.Tayyaba@hotmail.com).

Muhammad Waseem Ashraf and Muhammad Imran are with the Department of Physics (Electronics), GC University Lahore Pakistan (e-mail: Muhammad.Waseem.Ashraf@gmail.com).

Aneela Noreen is with the UET Lahore, Pakistan (e-mail: aneela_uet@hotmail.com). studies about 10,000 ear samples to demonstrate the inimitability of human ears [1]. Reference [2] used a method to identify the region of ear from a 3D image. They used two step algorithm with model template structure and online recognition. Reference [3] developed an ear recognition system by fusing SIFT features of color segmented slice regions of an ear. Reference [4] reported kernel independent component analysis (KICA) and support vector machine (SVM) with Gaussian radial basis function (GRBF) for ear feature extraction and classification. Reference [5] used a model based scheme for ear feature extraction. Reference [6] used wavelets transform for ear recognition and results achieved were up to $94.3 \%$. Reference [7] used a multimodal biometric system for recognition of human faces and ears by means of Eigen faces and Eigen ears. Reference [8] developed two 3D ear detection systems by structure from motion (SFM) and shape from shading (SFS) methods. By interpolation of edge and ravine, they performed segmentation of the ear section. Reference [9] reported a study of ear biometrics by using various methods like Eigen ear, principle components analysis (PCA), Hausdorff matching and ICP matching .Ear images were mapped to energy field by applying feature extraction using force field [10]. The wavelet approach was developed by using the log Gabor filter [11]. Reference [12] used the feature level fusion technique for ear biometric system. The process of template matching computes the degree of similarity between two images. One image is called template image and second is called the input image. Various algorithms and methods have been used for template matching. Reference [13] reported a template matching algorithm. The exact Legendre moment invariants were employed by cross-correlation for image recognition.

In this research work, the authors present a novel ear identification system for security applications. The proposed system consists of three modules that are database module, image processing module and identification module. The system extracts valuable information and removes noise from the processed images to improve the accuracy of the results. Testing has been performed to show the accuracy and the efficiency of the ear identification system. The system has been tested for 24 ear images by using template matching process. Experimental results show that the system is highly accurate and robust. This system is easily applicable in the police stations at the remote areas.

\section{SYSTEM DESCRIPTION}

The ear identification system consists of three modules that are database module, image processing module and identification module as shown in Fig. 1. 


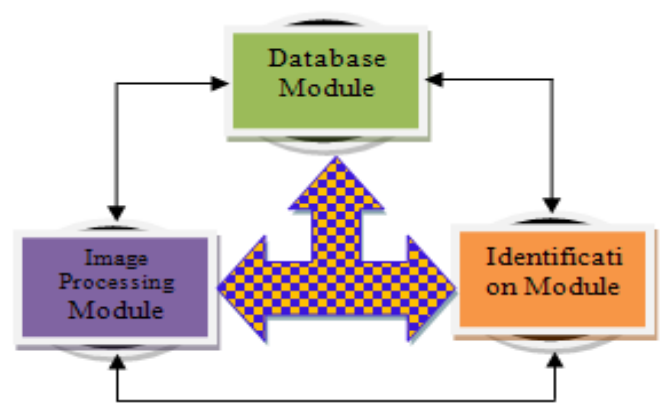

Fig. 1. Modules of ear identification system.

The database module consists of all information and generated results of the processed images. The image processing module extracts useful information from the images and removes noise that is present in the images. The identification module performs image assessment by template matching and makes decision about similarity or difference between images. The final decision is sent to report generator that provides the final result of ear identification process. The detailed block diagram of ear identification system is shown in Fig. 2.

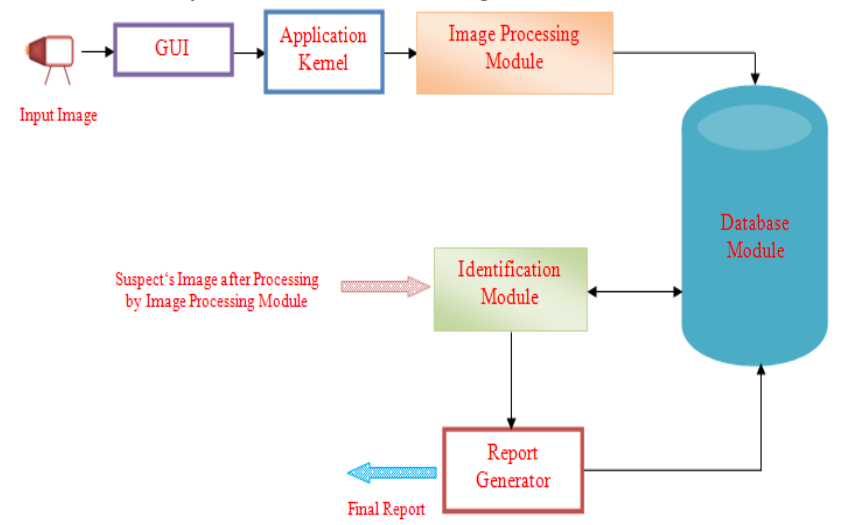

Fig. 2. Block diagram of ear identification system.

\section{Methodology}

The proposed ear identification system has different phases like image acquision, image enhancement, gray scale conversion, segmentation, thresholding, thinning, and template matching. In image acquision phase, the system aquire the image and load it in application framework as shown in Fig. 3.

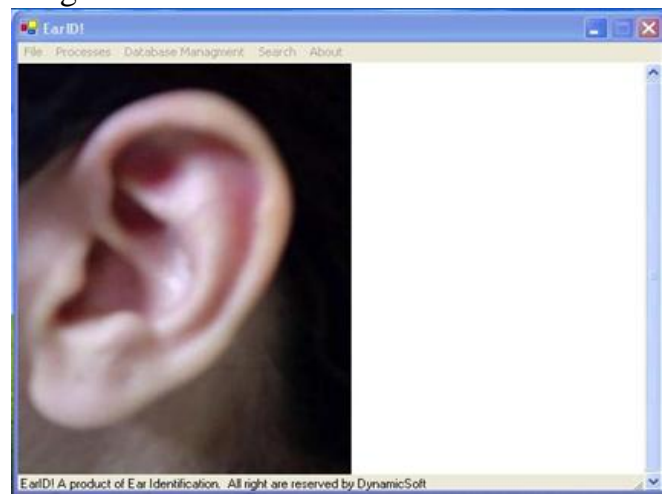

Fig. 3. Image loading in application framework.

In next phase, the enhancement of image is done. It improves the contrast, visibility and quality of ear image as shown in Fig. 4.

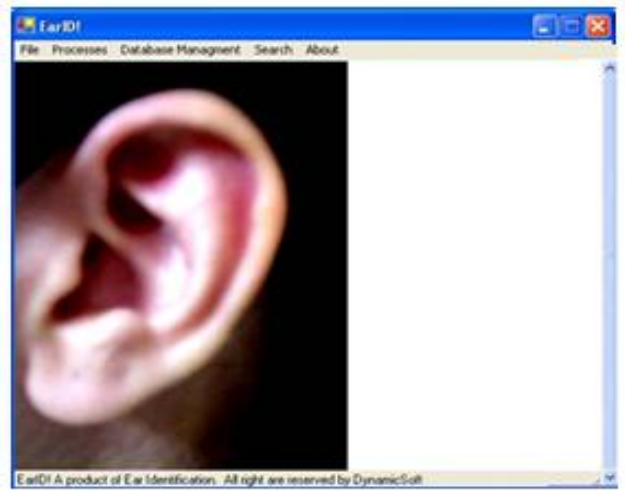

Fig. 4. Enhancement of image.

After this, the image is converted into gray scale in which the value of each pixel is converted into a single sample by using gray scale conversion $(G S C)$ equation as given below:

$$
G S C=0.299 R+0.587 G+0.144 B
$$

The image contains only intensity values after gray scale conversion. The image result of gray scale conversion is shown in Fig. 5.

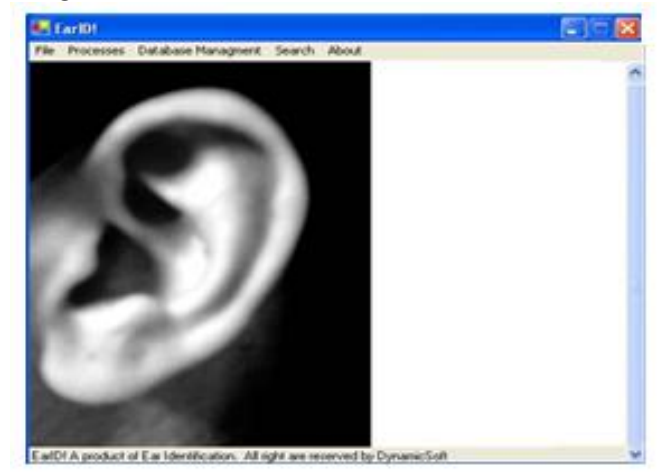

Fig. 5. Gray scale conversion of image.

In next phase, segmentation of image has been performed. The first step of segmentation is the histogram equalization. The process histogram equalization has been employed by using following equation:

$$
H(x, y)=C \times G(x, y)+B
$$

where $H(x, y)$ is the output image, $C$ is the contrast constant, $G(x, y)$ is the gray scale image and $B$ is the brightness constant.

By histogram equalization, the intensity vision of the image is achieved and approaching into the image composition is done. The results of histogram equalization give the information about the intensity division and contrast of the image as shown in Fig. 6.

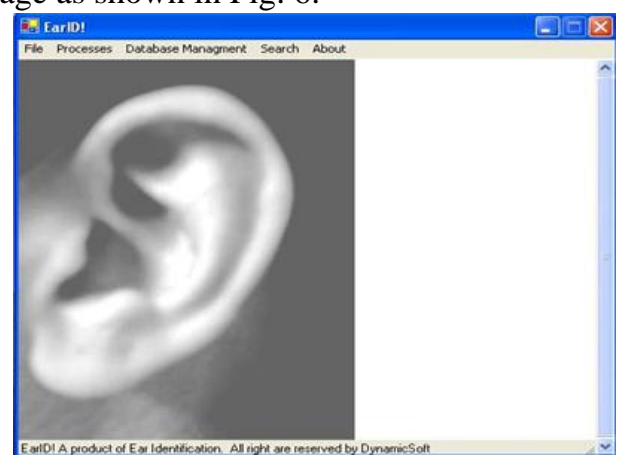

Fig. 6. Histogram equalization of image. 
Most of the information present in an image is hold by the edges. Edges give information about the object position, shape and size. The image result of edge detection is shown in Fig. 7.

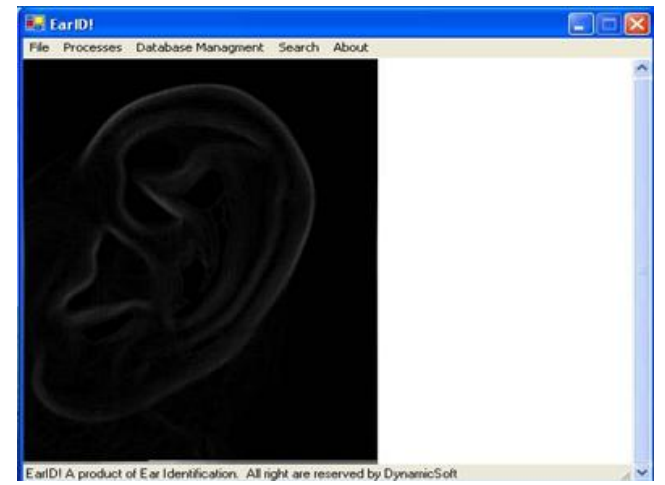

Fig. 7. Edge detection of image.

The thresholding of image has been used along with edge detection to accentuate the edges of images. The resultant image after applying thresholding is shown in Fig. 8.

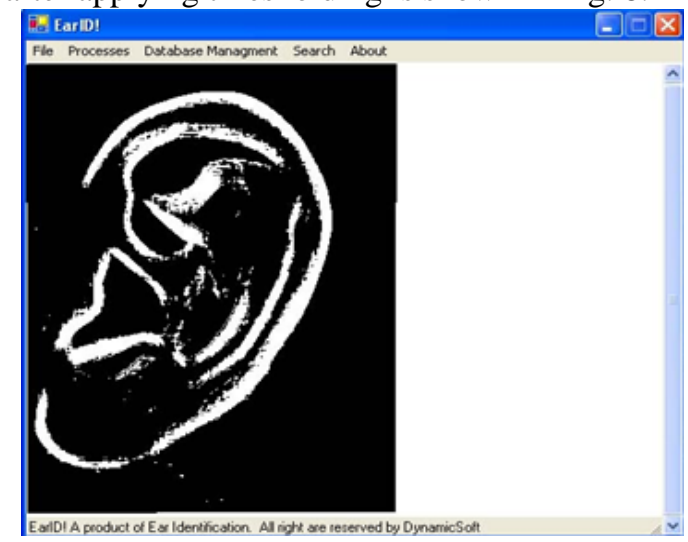

Fig. 8. Image thresholding.

After thresholding, thinning has been applied on the resultant image. The process of thinning converts the dark points into white with the length of pattern edges and results into thin lines. Thinning has removed the selected foreground pixels from the image by applying following equation [14]:

$$
\operatorname{Thin}(A)=A \cap \neg \mathrm{H}-\mathrm{M}(A)
$$

where $A$ represents the input image, and H-M is the hit and miss function.

The resultant image after thinning has been shown in Fig. 9.

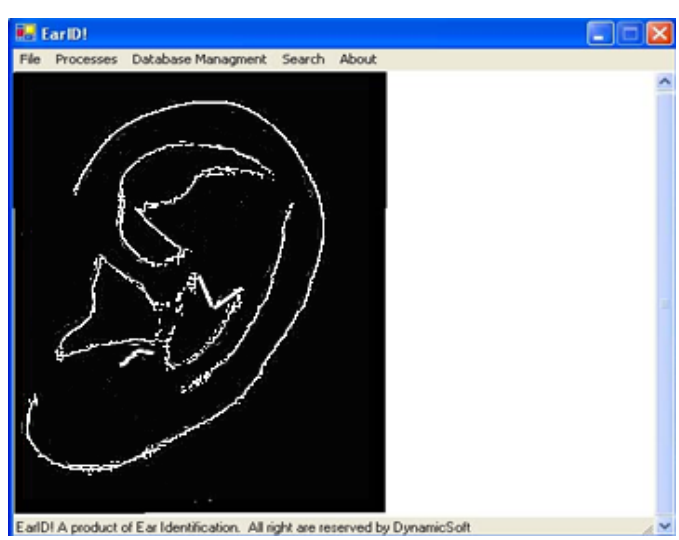

Fig. 9. Image result of thinning.

After thinnning, template matching has been performed using the equation of correlation as shown below [15]:

$$
R(x, y)=\sum_{s=0}^{N} \sum_{t=0}^{M} F_{t}(s, t) A(x+s, y+t)
$$

where $R(x, y)$ is the output image, $F 1(s, t)$ is the template and $A$ represents input image (suspect's image).

The output value of template matching operation will be highest at those pixel points where the image arrangement matches with the template. Here the large values of image are multiplied by large values of template. A set of templates of each ear for matching process has been used that includes the lobe shape template, normalized length template (normalized length has been obtained by dividing ear width with ear height), tragus shape template, helical rim template, antihelical fold template, concha shape template, triangular fossa template and scaphoid fossa template. Some of these templates are shown in Fig. 10.
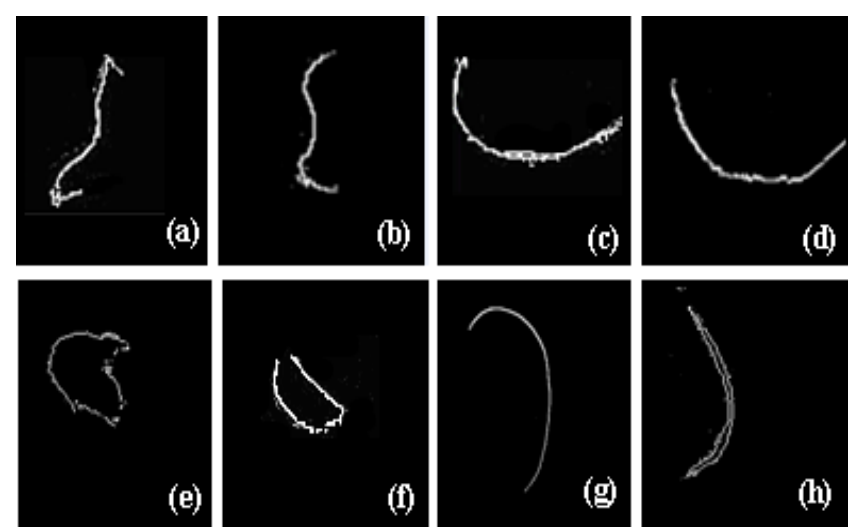

Fig. 10. Templates for matching process (a) Tragus template 1 (b) Tragus template 2 (c) Lobe template 1 (d) Lobe shape template 2 (e) Concha shape template (f) Triangular fossa template (g) Helical rim template (h) Antihelical fold template.

\section{RESUlTS AND DISCUSSION}

The efficiency of the developed ear identification system has been tested using the templates on ear images. The resultant output images consist of black and white pixels. The white pixels represent the similarity value and the black pixels represent the difference value between the template and original image. If there are whiter pixels present than black pixels, it shows that the similarity or matching between template and input image (suspect image) is more precise. But if black pixels are more than white pixels in output image it shows that the matching is not precise and there is a difference between two images. The output images divulge the resultant image and location of the greatest match between the template and the original image (input image). When the templates of same ear image were used then the result of template matching employed the similarity between the image and templates as shown in Fig. 11(a) and Fig. 11 (c). But when templates of different ear image were used then the results showed that the input images had no similarity with the templates as shown in Fig. 11 (b) and Fig. 11 (d). 

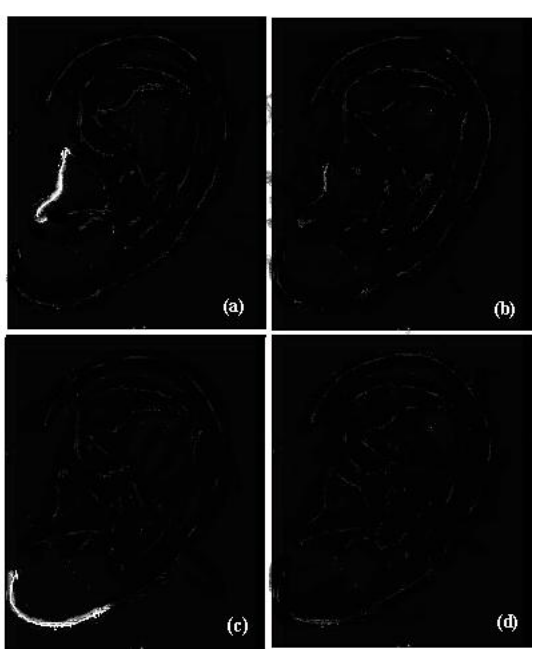

Fig. 11. Image results of template matching (a) Result of tragus template1 (b) Result of tragus template 2 (c) Result of lobe template 1 (d) Result of lobe template 2 .

\section{CONCLUSION}

In this study, a novel ear identification system has been presented for security applications in remote areas. The proposed system consists of three modules that are database module, image processing module and identification module. The system extracts useful information and removes noise from the input images by image processing module. The data base module holds all information and results of processed images. The identification module performs the image assessment with the record present in database using template matching. The system has been tested for 24 ear images by using templates. Experimental results show that the system is 98\% accurate and demonstrates the higher degree of robustness and accuracy of the matching process.

\section{REFERENCES}

[1] A. Iannarelli, "Ear identification, forensic identification series," Paramont Publishing Company, Fremont, California, 1989.

[2] H. Chen and B. Bhanu, "Contour matching for 3D ear recognition," Seventh IEEE Workshops on Application of Computer Vision, pp. 123-128, 2005.

[3] D. R. Kisku, H. Mehrotra, P. Gupta, and J. K. Sing, "SIFT-based ear recognition by fusion of detected keypoints from color similarity slice regions," International Conference on Advances in Computational Tools for Engineering Applications, pp. 380-385, 2009.

[4] W. H. Lei, W. Qian, S. H. Jun, and H. L. Yan, "Ear identificaton based on KICA and SVM," Global Congress on Intelligent Systems, pp. 414-417, 2009.

[5] E. Jeges and L. Mate, "Model-based human ear identification," Automation Congress, pp. 1-6, 2006.

[6] M. Ali, M. Y. Javed, and A. Basit, "Ear recognition using wavelets," Proceedings of Image and Vision Computing New Zealand, pp. 83-86, 2007.

[7] A. A. Darwish, R. AbdElghafar, and A. F. Ali, "Multimodal face and ear images," Journal of Computer Science, vol.5, no. 5, pp. 374-379, 2009.

[8] M. A. Mottaleb and J. Zhou, "A system for ear biometrics from face profile images," Special Issue on Biometrics: ICGST International Journal on Graphics, Vision and Image Processing, 2006.

[9] P. Yan and K. W. Bowyer, "An automatic 3D ear recognition system," Third International Symposium on 3D Data Processing, Visualization and Transmission, pp. 326-333, 2006.

[10] D. J. Hurley, M. S. Nixon, and J. N. Carter, "Force field energy functionals for image feature extraction," Proceedings of Proc. 10th British Machine Vision Conference, pp. 604-613, 1999.

[11] B. A. Zavar and M. S. Nixon, "Robust log-gabor filter for ear biometrics," 19th International Conference on Pattern Recognition, pp. $1-4,2008$.

[12] G. S. Badrinath and P. Gupta, "Feature level fused ear biometric system," Seventh International Conference on Advances in Pattern Recognition, pp. 197-200, 2009.

[13] K. M. Hosny, "Robust template matching using orthogonal legendre moment invariants," Journal of Computer Science, vol. 6, pp. 1054-1058, 2010.

[14] R. Gonzalez and R. Woods, "Digital image processing," Addison-Wesley Publishing Company, pp. 518 - 548, 1992.

[15] H. R. Myler and A. R. Weeks, "The pocket handbook of imaging processing algorithms in C," Department of Electrical and Computer Engineering, University of Central Florida, Orlando Florida.1993. 